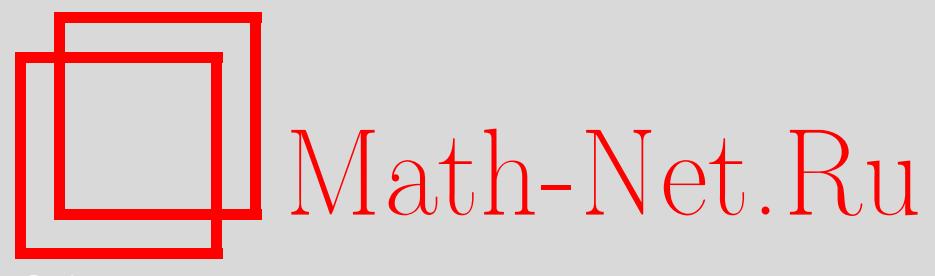

Ш. Х. Ганцев, Р. Н. Бахтизин, М. В. Франц, К. Ш. Ганцев, Опухолевый рост и возможности математического моделирования системных процессов, Вестн. Сам. гос. техн. ун-та. Сер. Физ.мат. науки, 2019, номер 1, 131-151

DOI: https://doi.org/10.14498/vsgtu1661

Использование Общероссийского математического портала MathNet.Ru подразумевает, что вы прочитали и согласны с пользовательским соглашением

http: //www . mathnet.ru/rus/agreement

Параметры загрузки:

IP : 54.84 .234 .179

26 апреля 2023 г., 16:20:46

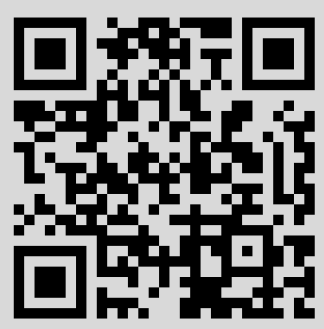


Вестн. Сам. гос. техн. ун-та. Сер. Физ.-мат. науки. 2019. Т. 23, № 1. С. $131-151$ ISSN: 2310-7081 (online), 1991-8615 (print)

УДК 517.958:57

\title{
Опухолевый рост и возможности математического моделирования системных процессов
}

\author{
Ш. Х. Ганцев ${ }^{1}$, Р. Н. Бахтизин ${ }^{2}$, М. В. Франи ${ }_{3}^{3}$ К. Ш. Ганцев \\ 1 Башкирский государственный медицинский университет, \\ Россия, 450000, Уфа, ул. Ленина, 3. \\ 2 Уфимский государственный нефтяной технический университет, \\ Россия, 450062, Уфа, ул. Космонавтов 1. \\ 3 Уфимский государственный авиационный технический университет, \\ Россия, 450008, Уфа, ул. К. Маркса, 12.
}

\section{Аннотация}

В работе обсуждаются вопросы применения математического моделирования к исследованию процесса опухолевого роста и проблеме оптимизации лечения онкологических заболеваний. Приводится структурированный обзор работ отечественных и зарубежных авторов, посвященных этой проблематике. Обсуждается важность представлений о жизненном цикле клетки в понимании опухолевого процесса и механизмов лечения онкологических заболеваний, связанная прежде всего с тем, что применяемые методы лечения, в частности, химиотерапия и лучевая терапия, действуют как на нормальные, так и на опухолевые клетки, находящиеся в определенных стадиях жизненного цикла, и не поражают клетки в других стадиях. Приводится описание жизненного цикла клетки и механизмов, в норме обеспечивающих сохранение и восстановление нормальной плотности клеточной популяции, приводится граф стадий и переходов клетки. Предлагается математическая модель поддержания пролиферативного гомеостаза в клеточной популяции, которая учитывает гетерогенность клеточных популяций по стадиям жизненного цикла. Модель представляет собой систему дифференциальных уравнений с запаздыванием. Условия стационарности позволяют определить значения параметров модели, присущих нормальной жизнедеятельности клеточной популяции. В работе приводятся результаты вычислительного

\section{Научная статья}

ə () (i) Контент публикуется на условиях лицензии Creative Commons Attribution 4.0 International (https://creativecommons.org/licenses/by/4.0/deed.ru)

\section{Образец для цитирования}

Ганцев Ш. Х., Бахтизин Р. Н., Франц М. В., Ганцев К. Ш. Опухолевый рост и возможности математического моделирования системных процессов // Becmн. Сам. гос. техн. ун-та. Сер. Физ.-мат. науки, 2019. Т. 23, № 1. С. 131-151. doi: 10.14498/vsgtu1661.

\section{Сведения об авторах}

Шамиль Ханафиевич Ганиев (D) https://orcid.org/0000-0003-2047-963X доктор медицинских наук, профессор; заведующий кафедрой; каф. онкологии; e-mail:prfg@mail.ru

Рамиль Назифович Бахтизин; доктор физико-математических наук, профессор; ректор; e-mail: rektor@rusoil.net

Марина Валерьевна Франи, (1) https://orcid.org/0000-0002-5324-2463 кандидат технических наук; доцент; каф. экономики предпринимательства; e-mail: tan-Marina@mail.ru

Камиль Шамилевич Ганцев (1) https://orcid.org/0000-0002-7562-5684 доктор медицинских наук, профессор; профессор; каф. онкологии;

e-mail: Gantseff@mail.ru 
эксперимента, в котором исследуется процесс восстановления плотности клеточной популяции в случае массовой гибели клеток. Как показывает эксперимент, после гибели клеток происходит восстановление плотности клеток в разных стадиях до нормальных значений, что соответствует представлениям о пролиферативном гомеостазе в клеточных популяциях.

Ключевые слова: опухолевый рост, пролиферативный гомеостаз, жизненный цикл клетки, клеточная кинетика.

Получение: 18 ноября 2018 г. / Исправление: 22 января 2019 г. /

Принятие: 27 января 2019 г. / Публикация онлайн: 11 марта 2019 г.

Введение. Математическое моделирование процесса опухолевого роста. Онкологические заболевания являются глобальной проблемой, и для ее решения необходимы подходы мультидисциплинарного плана. Следует констатировать, что остроту проблеме придает повсеместный рост заболеваемости, и особенно рост смертности. Так, по прогнозам исследователей, в 2018 году в мире будет зарегистрировано 18.1 млн онкологических больных, при этом прогнозируется, что 9.6 млн человек скончается от онкологических заболеваний [1].

Накопленные к настоящему времени данные о патогенезе рака требуют своего обобщения и теоретического анализа. В этом процессе важную роль могут сыграть математические модели, описывающие основные причинноследственные связи, определяющие динамику процесса опухолевого роста. Такие модели могут быть полезны для проверки гипотез и теорий о механизмах формирования тех или иных эффектов, наблюдающихся при онкологических заболеваниях, а также для обобщения накопленного экспериментального материала. В ряде случаев математические модели могут заменять эксперименты, проведение которых не представляется возможным. С практической точки зрения использование метода математического моделирования в онкологии открывает возможности для количественного и временного прогнозирования течения онкологического заболевания и влияния различных видов лечебных воздействий на организм пациента на различных уровнях: на уровне отдельных клеток, тканей, организма в целом.

На современном этапе математическое моделирование опухолевого роста и влияния на этот процесс с помощью различных видов лечения (хирургического, химиотерапевтического, радиологического, иммунологического, гормонального и т.п.) является довольно активно развивающимся направлением исследований.

Ряд исследований посвящен изучению динамики «естественного» роста опухолей и формирования специфических эффектов, порождаемых этим процессом. Наиболее ранние работы имели своей целью математическое описание наблюдаемой динамики опухолевого процесса $[2,3]$ как баланса между делением и гибелью клеток в опухолевой популяции. После появления теории о том, что иммунная система организма способна угнетать и даже совсем предотвращать опухолевый рост на ранних стадиях, были разработаны модели, описывающие взаимодействие опухоли с иммунной системой с разной 
степенью детализации описания иммунного ответа [4-7]. Всесторонний обзор эволюции моделей этой направленности дан в работе [8].

Так как рост опухоли зачастую происходит быстрыми темпами, в опухолевых тканях не успевает сформироваться в полной мере присущая нормальным тканям система кровоснабжения, обеспечивающая доставку кислорода и питательных веществ к клеткам. Поэтому уровень кислорода и питательных веществ в опухолевых тканях отличается от этих параметров в нормальных тканях. Это приводит к тому, что в опухолевых тканях наблюдаются участки некроза, т.е. скопления опухолевых клеток, погибших вследствие недостатка кислорода и питательных веществ. Математические модели данных процессов представлены в работах [9-11]. Так, в работе [9] предложена модель диффузии кислорода в опухоль сферической формы, позволяющая предсказывать размер некротического ядра и зону гипоксии. Показано, что результаты моделирования хорошо согласуются с экспериментальными данными. В работах $[10,11]$ обсуждаются модели диффузии кислорода в опухолях различной формы (прежде всего сфероидной и цилиндрической), различного размера и источника кровоснабжения. С практической точки зрения эти работы важны для прогнозирования результатов лучевой терапии, эффект воздействия которой зависит от концентрации кислорода в опухоли.

Генетическая неоднородность опухолевых клеток, порождаемая высоким уровнем генетических мутаций в опухолевой популяции в процессе развития онкологического заболевания, является огромной проблемой, так как приводит к резистентности опухолей к различным видам лечения. Наиболее ранние математические модели, посвященные этой особенности опухолевого роста, рассматривали точечные мутации [12]. В дальнейшем были предложены модели, описывающие стохастические многоступенчатые процессы мутаций опухолевых клеток [13-16]. Относительно новым направлением исследований является разработка моделей, в которых процесс мутации клеток в опухоли рассматривается не как ступенчатый или дискретный, а как непрерывный $[17,18]$. Важным моментом в тематике генетической неоднородности опухолевой популяции является факт, заключающийся в том, что приводящие к резистентности генетические мутации могут быть как спонтанными, так и индуцированными применявшимся ранее лечением. Поэтому ряд разработок в этой области связан с моделированием мутаций, возникающих под воздействием полихимиотерапевтического лечения [19,20].

Радиологический метод очень давно используется при лечении онкологических заболеваний. В работе [21] предложена математическая модель, описывающая сосуществование нормальной и опухолевой клеточных популяций под влиянием периодической лучевой терапии. Отмечается, что в работах [22,23], которые посвящены «исследованию воздействия ионизирующей радиации на жизненно важные системы организма млекопитающих», разработаны «математические модели кроветворения, тонкого кишечника, гуморального иммунитета, которые на количественном уровне воспроизводят динамику этих систем у млекопитающих, подвергающихся острому и хроническому облучению в широких диапазонах доз и мощностей доз» [21]. В практическом смысле предлагаемые модели могут быть полезны для прогнозирования эффектов и побочных явлений при применении радиологического метода лечения онкологических заболеваний. 
Химиотерапевтическое лечение является в настоящее время основным методом терапии в онкологии, поэтому ряд работ посвящен моделированию влияния этого вида лечения на опухолевый рост. В работе [24] исследуется задача оптимизации режима введения одного химиопрепарата таким образом, чтобы максимально уменьшить размер опухоли и при этом не допустить снижения числа клеток в нормальной клеточной популяции ниже определенного уровня. В работах [25,26] исследуется математическая модель, описывающая динамику концентрации нормальных, опухолевых и иммунных клеток под влиянием химиотерапевтического лечения.

Иммунологическое лечение, направленное на стимуляцию реакции иммунной системы на опухолевый рост, является не таким широко используемым на практике методом, как химиотерапия и лучевая терапия. Однако этот метод потенциально весьма привлекателен в связи с тем, что он гораздо менее токсичен по отношению к нормальным тканям. В работе [27] исследуется математическая модель влияния иммунотерапевтического лечения на развитие меланомы кожи и решается задача оптимизации плана лечения (т.е. доз и режимов введения препаратов). Целевая функция в рассматриваемой оптимизационной задаче - минимизация вероятности истощения $T$-клеток на различных стадиях развития болезни. В работе [28] предложена модель, описывающая динамику опухолевого роста под влиянием иммунотерапии, которая строится на базе агентно-ориентированного подхода.

Перспективным направлением в терапии рака является вирусотерапия, т.е. использование для лечения онкологических заболеваний вирусов, специфически поражающих опухолевые клетки. Существует несколько механизмов поражения раковых клеток онколитическими вирусами:

- многократная репликация вируса внутри раковой клетки, приводящая к ее гибели;

- выработка цитотоксического протеина, повреждающего раковые клетки;

- стимуляция иммунной системы организма в связи с воспалительным процессом, индуцированным вирусом.

Математическому моделированию вирусотерапии злокачественных опухолей посвящена работа [29], в которой предложена и исследована математическая модель, описывающая взаимодействие онколитического вируса и опухолевых клеток и учитывающая первый из перечисленных механизмов поражения раковых клеток.

В настоящей работе предлагается математическая модель поддержания пролиферативного гомеостаза в клеточной популяции, основанная на представлениях о жизненном цикле клетки. Такое представление важно для моделирования радиологического и химиотерапевтического воздействия на нормальные и опухолевые ткани, так как современные противоопухолевые препараты оказывают избирательное воздействие на определенные структуры клетки в разные этапы жизненного цикла, а чувствительность клеток к облучению также зависит от этих факторов. В работе приводится описание жизненного цикла клетки и механизмов, в норме обеспечивающих сохранение и восстановление нормальной плотности клеточной популяции, которые используются как основа для разработки математической модели. Подробно описывается модель пролиферативного гомеостаза в нормальной клеточной 
популяции, определяются характеристики стационарного режима, приводятся результаты вычислительного эксперимента и их сопоставление с экспериментальными данными.

\section{1. Жизненный цикл клетки и регуляция размножения клеток} в норме. Многоклеточный живой организм состоит из тканей, т.е. сформировавшихся в процессе эволюции клеточных популяций и внеклеточных структур. Клетка является элементарной структурно-функциональной единицей организма. Несмотря на многообразие типов клеток, значительные их различия по форме, размерам, выполняемым функциям, все они имеют похожее строение. Функционирование многоклеточного организма определяется способностью клеток реагировать на изменение внешней среды и взаимодействовать между собой. Поведение клетки определяется сигналами, получаемыми из окружающей среды. Под влиянием определенных сигналов клетка способна, например, начать процесс деления или, наоборот, выйти из цикла деления и перестроиться в специализированную клетку, способную выполнять определенную функцию в организме, и даже включить программу самоубийства (апоптоза). «Органами чувств» клетки являются поверхностные рецепторы, способные «узнавать» то или иное биологически активное вещество.

В ходе эволюции в живых организмах выработались компенсаторные механизмы, направленные на восстановление нарушенных функций. При утрате части клеток в тканях запускается процесс пролиферации, т.е. деления клеток для замещения утраченных. При этом эти процессы протекают таким образом, чтобы в итоге вернуть присущие взрослому организму соотношения размеров и масс различных органов. При этом используется концепция пролиферативного гомеостаза, согласно которой повышение количества делящихся клеток приводит к включению механизмов, препятствующих дальнейшей пролиферации.

Пролиферативный гомеостаз в организме в значительной мере поддерживается кейлонной ингибиторной системой. Кейлоны - биологически активные вещества, вырабатываемые в норме зрелыми клетками, контролирующие процессы деления клеток по принципу обратной связи. При утрате зрелых клеток в результате какого-либо повреждения концентрация кейлонов понижается и запускается процесс пролиферации. По мере восстановления необходимой плотности зрелых клеток концентрация кейлонов повышается, что приводит к снижению интенсивности деления клеток.

Онкологическое заболевание представляет собой появление в организме и размножение клона или нескольких клонов клеток, пролиферативная активность которых не подчиняется регуляторным механизмам, присущим нормальным клеточным популяциям. Жизненный цикл клетки включает следующие основные этапы [30]:

- Стадия роста $\left(\mathrm{G}_{1}\right)$. В эту стадию клетки попадают сразу после деления материнской клетки на две дочерние. Во время пребывания клетки в этой стадии происходит увеличение цитоплазмы, синтез и накопление веществ, необходимых для удвоения ДНК. К окончанию этой стадии клетка готова к тому, чтобы начать новый цикл деления. Клетка в стадии $\mathrm{G}_{1}$ пребывает фиксированное время.

- Стадия покоя $\left(\mathrm{G}_{0}\right)$. В эту стадию клетка попадает из стадии роста. Если 
в популяции клеток наблюдается недостаток высокоспециализированных дифференцированных (зрелых) клеток, то клетки, находящиеся в стадии $\mathrm{G}_{0}$, могут переходить в стадии деления и зрелости. Важно, что опухолевые клетки также способны задерживаться в стадии покоя и затем вновь возвращаться в стадию деления.

- Стадия деления $\left(S-G_{2}-M\right)$. В эту стадию попадают клетки из стадии покоя при недостатке высокоспециализированных дифференцированных клеток. Клетка в стадии деления пребывает фиксированное время. Стадия деления разделяется на три фазы:

- S - синтетический период, характеризующийся удвоением молекул ДНК;

- $\mathrm{G}_{2}$ - постсинтетический период, в котором происходит интенсивный синтез белков;

- $\mathrm{M}$ - митоз, в котором хромосомы расщепляются, ядро раздваивается, цитоплазма делится пополам и в результате образуются две дочерние клетки.

- Стадия зрелости (дифференцировки) (Z). В эту стадию клетки попадают из стадии покоя при недостатке дифференцированных клеток в популяции. После попадания в это состояние клетка трансформируется в дифференцированную, т.е. способную выполнять специфическую функцию в организме, свойственную данному виду клеток. При этом она теряет способность делиться. Выполняя свою функцию в организме, она изнашивается и, в конечном счете, погибает.

Граф стадий и переходов клетки между стадиями жизненного цикла приведен на рис. 1. Внутри кружков приведены идентификационные номера стадий, которые будут использоваться в математической модели.

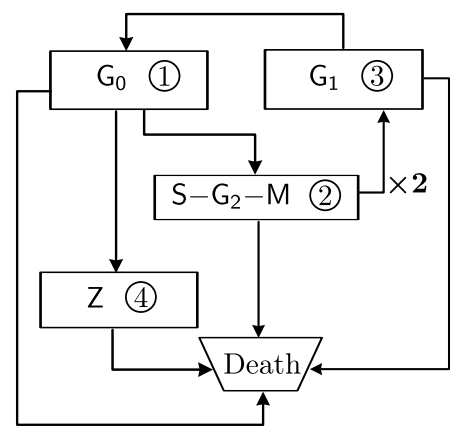

Рис. 1. Граф стадий и переходов клетки между стадиями жизненного цикла

[Figure 1. The cell cycle graph and transition between stages]

Примечательно, что современные противоопухолевые препараты оказывают избирательное воздействие на определенные структуры клеток в разные этапы жизненного цикла: антиметаболиты и дактиномицин действуют на клетки в S-фазе, блеомицин эффективен в $\mathrm{G}_{2}$-фазе, винкристин действует в М-фазе. ${ }^{1}$ Важно и то, что все клетки, находящиеся в стадии покоя, в том числе и опухолевые, нечувствительны к действию противоопухолевых химиопрепаратов и к лучевому воздействию. Такие покоящиеся клетки называются

\footnotetext{
${ }^{1}$ На основании данных портала «Справочник лекарств РЛС ${ }^{\circledR}$ 》 (http://www.rlsnet.ru).
} 
клоногенными; они могут быть источником рецидивов опухолей.

С учетом важности представлений о жизненном цикле клетки в понимании механизмов опухолевого роста в данной работе разрабатывается динамическая модель пролиферативного гомеостаза, являющаяся развитием наших более ранних исследований $[31,32]$. В дальнейших планах авторов - модификация этой модели для описания процесса опухолевого роста и воздействия на этот процесс различных видов лечения.

2. Математическая модель поддержания пролиферативного гомеостаза в нормальной клеточной популяции. Для компактности описания модели поставим в соответствие каждому этапу жизненного цикла идентификационный номер (см. рис. 1): 1 - стадия покоя, 2 - стадия деления, 3 - стадия роста, 4 - стадия зрелости. Введем следующие обозначения:

- $L_{i}(t)$ - плотность клеток в $i$-той стадии жизненного цикла;

- $\tau_{i}$ - средняя длительность жизни клетки в $i$-той стадии жизненного цикла;

- $d_{i}$ - длительность $i$-той стадии жизненного цикла $(i=1,3)$;

- $h$ - доля клеток в 4-й стадии жизненного цикла в стационарном состоянии;

- $L^{\mathrm{st}}$ - общая плотность клеток в стационарном состоянии;

- $b$ - параметр, определяющий интенсивность выхода клеток из 1-й стадии жизненного цикла;

- $a$ - параметр, характеризующий соотношение между интенсивностями переходов клеток из 1-й стадии во 2-ю и 4-ю стадии в стационарном состоянии.

Динамика плотности клеток на каждом этапе жизненного цикла описывается системой дифференциальных уравнений с запаздыванием, которая выписана ниже.

Уравнение

$$
\begin{aligned}
\frac{d L_{1}(t)}{d t}=2 p_{12}\left(t-d_{2}-d_{3}\right) L_{1}\left(t-d_{2}\right. & \left.-d_{3}\right) \phi_{1}- \\
& -p_{12}(t) L_{1}(t)-p_{14}(t) L_{1}(t)-\frac{L_{1}(t)}{\tau_{1}}
\end{aligned}
$$

описывает изменение плотности клеток в 1-й стадии жизненного цикла. Плотность клеток в этой стадии увеличивается за счет притока клеток из 3-й стадии, уменьшается за счет перехода клеток из 1-й стадии во 2-ю и 4-ю, а также гибели клеток в 1-й стадии. Компонент $\phi_{1}$, рассчитываемый по формуле

$$
\phi_{1}=\exp \left(-\frac{d_{2}}{\tau_{2}}\right) \exp \left(-\frac{d_{3}}{\tau_{3}}\right)
$$

позволяет учесть возможность гибели клеток при прохождении 2-й и 3-й стадий.

Изменение плотности клеток во 2-й стадии описывается уравнением

$$
\frac{d L_{2}(t)}{d t}=-p_{12}\left(t-d_{2}\right) L_{1}\left(t-d_{2}\right) \phi_{2}+p_{12}(t) L_{1}(t)-\frac{L_{2}(t)}{\tau_{2}}
$$

Увеличение плотности клеток во 2-й стадии происходит за счет перехода клеток из 1-й стадии во 2-ю, уменьшение - за счет перехода клеток 2-й стадии в 
3-ю и гибели клеток во 2-й стадии. Компонент $\phi_{2}$, рассчитываемый по формуле

$$
\phi_{2}=\exp \left(-\frac{d_{2}}{\tau_{2}}\right)
$$

позволяет учесть возможность гибели клеток во время прохождения 2-й стадии.

\section{Уравнение}

$$
\frac{d L_{3}(t)}{d t}=2 p_{12}\left(t-d_{2}\right) L_{1}\left(t-d_{2}\right) \phi_{2}-2 p_{12}\left(t-d_{2}-d_{3}\right) L_{1}\left(t-d_{2}-d_{3}\right) \phi_{1}-\frac{L_{3}(t)}{\tau_{3}}
$$

описывает изменение плотности клеток в 3-й стадии жизненного цикла. Плотность клеток в этой стадии увеличивается за счет перехода клеток из 2-й стадии в 3-ю с удвоением, уменьшается за счет перехода клеток из 3-й стадии в 1-ю, а также гибели клеток в 3-й стадии.

Изменение плотности клеток в 4-й стадии определяется уравнением

$$
\frac{d L_{4}(t)}{d t}=p_{14}(t) L_{1}(t)-\frac{L_{4}(t)}{\tau_{4}} .
$$

Плотность клеток в 4-й стадии увеличивается за счет перехода клеток из 1-й стадии в 4-ю, уменьшается из-за гибели клеток в 4-й стадии.

Уравнения

$$
\begin{gathered}
p_{12}(t)=\exp \left[-a\left(\frac{L_{1}(t)+L_{2}(t)+L_{3}(t)}{(1-h) L^{\mathrm{st}}}\right)^{2}\right] \exp \left[-b\left(\frac{L_{4}(t)}{h L^{\mathrm{st}}}\right)^{2}\right], \\
p_{14}(t)=\left(1-\exp \left[-a\left(\frac{L_{1}(t)+L_{2}(t)+L_{3}(t)}{(1-h) L^{\mathrm{st}}}\right)^{2}\right]\right) \exp \left[-b\left(\frac{L_{4}(t)}{h L^{\mathrm{st}}}\right)^{2}\right]
\end{gathered}
$$

описывают интенсивности переходов клеток из 1-й стадии во 2-ю и 4-ю соответственно. Интенсивность выхода клеток из 1-й стадии зависит от соотношения плотности клеток в 4-й стадии жизненного цикла с их плотностью в норме. Выбор между 2-й и 4-й стадиями зависит от соотношения плотности клеток, способных к делению (т.е. клеток в 1-3 стадиях), с их плотностью в норме. Чем меньше плотность клеток, способных к делению, тем большая часть клеток, выходящих из 1-й стадии, переходит во 2-ю стадию.

Условия стационарности, описываемые уравнениями

$$
\begin{gathered}
\frac{d L_{i}(t)}{d t}=0, \quad i=1,2,3,4, \\
\frac{L_{1}^{\mathrm{st}}+L_{2}^{\mathrm{st}}+L_{3}^{\mathrm{st}}}{(1-h) L^{\mathrm{st}}}=1, \quad \frac{L_{4}^{\mathrm{st}}}{h L^{\mathrm{st}}}=1,
\end{gathered}
$$

определяют нормальную жизнедеятельность клеточной популяции, в которой поддерживается стабильная плотность клеток во всех стадиях.

Решая систему уравнений (9), (10), удается получить характеристики стационарного режима:

$$
p_{12}^{\mathrm{st}}=\frac{h \tau_{1}+(1-h) \tau_{4}}{\tau_{1}\left[(1-h)\left(2 \phi_{1}-1\right) \tau_{4}-h\left(\tau_{2}\left(1-\phi_{2}\right)+2 \tau_{3}\left(\phi_{2}-\phi_{1}\right)\right)\right]},
$$




$$
\begin{gathered}
p_{14}^{\mathrm{st}}=p_{12}^{\mathrm{st}}\left(2 \phi_{1}-1\right)-\frac{1}{\tau_{1}} ; \\
L_{1}^{\mathrm{st}}=\frac{h L^{\mathrm{st}}}{\tau_{4} p_{14}^{\mathrm{st}}}, \quad L_{2}^{\mathrm{st}}=\tau_{2} p_{12}^{\mathrm{st}} L_{1}^{\mathrm{st}}\left(1-\phi_{2}\right), \\
L_{3}^{\mathrm{st}}=2 p_{12}^{\mathrm{st}} L_{1}^{\mathrm{st}} \tau_{3}\left(\phi_{2}-\phi_{1}\right), \quad L_{4}^{\mathrm{st}}=h L^{\mathrm{st}}
\end{gathered}
$$

и значения параметров $a$ и $b$ :

$$
a=\ln \left(1+\frac{p_{14}^{\mathrm{st}}}{p_{12}^{\mathrm{st}}}\right), \quad b=\ln \left(\frac{1}{p_{12}^{\mathrm{st}}+p_{14}^{\mathrm{st}}}\right) .
$$

3. Выбор значений для параметров модели. Несмотря на обилие работ, описывающих жизненный цикл клетки, далеко не всегда в них приводятся сведения, содержащие информацию количественного плана, более того, количественные оценки длительности отдельных этапов жизненного цикла клетки в разных источниках могут весьма сильно отличаться.

В своих экспериментах мы опирались на работу [33], в которой приведены следующие сведения о длительности этапов жизненного цикла:

- стадия $\mathrm{G}_{1}$ длится $12-24$ часов,

- фаза S длится около 5 часов,

- стадия $\mathrm{G}_{2}$ длится около 4 часов,

- фаза М длится около 1.5 часов.

Исходя из этого в вычислительном эксперименте были взяты следующие значения параметров: $d_{2}=10.5$ часов, $d_{3}=18$ часов.

В работе [33] также указывается, что организм взрослого человека теряет приблизительно 1-2\% клеток в день в результате их гибели. Соответственно, интенсивность гибели (вероятность погибнуть в течение дня) составляет приблизительно 0.015, следовательно, среднее время жизни составляет 66.67 дня, или 1600 часов. Поэтому в вычислительном эксперименте параметр $\tau_{4}=1600$ часов.

В работе [34], посвященной стволовым клеткам, указывается, что регионарные стволовые клетки взрослого организма, расположенные в тканях и органах, нуждаюшихся в обновлении, существуют и сохраняют свою способность к делению на протяжении всей жизни организма, поэтому длительность жизни клеток в стадиях покоя, деления и роста взята высокой, сравнимой с длительностью жизни организма в целом: $\tau_{1}=\tau_{2}=\tau_{3}=500000$ часов.

Доля зрелых клеток в популяции оценивалась исходя из следующих соображений. Известно, что митотический индекс (т.е. доля клеток в фазе митоза в тканях или клеточных культурах) весьма сильно колеблется в тканях разного вида: наиболее высокие его показатели характерны для росткового слоя кожи (0.7), верхушечной и боковой меристемы $(0.7)$, эпителия тонкого кишечника (0.78), клеток красного костного мозга (0.74), а наиболее низкие для скелетной мышечной ткани (0.0001) и нервной ткани (0.0001).

Учитывая, что фаза митоза занимает $5.2 \%$ клеточного цикла, и принимая для митотического индекса значение на уровне 0.3 , получаем, что доля клеток в клеточном цикле составляет примерно $5.7 \%$. Следовательно, доля зрелых клеток - примерно 0.943. Поэтому в вычислительном эксперименте был выбран параметр $h=0.943$. 
Плотность клеток в организме оценивалась на основе работы [35], в которой установлено, что в организме мыши весом 25 г приблизительно $3 \cdot 10^{9}$ клеток. Следовательно, плотность клеток в организме млекопитающего составляет приблизительно $1.2 \cdot 10^{8}$ клеток/г. Поэтому в вычислительном эксперименте был выбран параметр $L^{\mathrm{st}}=1.2 \cdot 10^{8}$.

4. Вычислительный эксперимент. Динамика восстановления плотности клеточной популяции в случае гибели части клеток. Приведем результаты моделирования изменения плотности клеток на различных этапах жизненного цикла клеток в случае гибели $20 \%$ зрелых клеток в клеточной популяции. Коэффициенты $\phi_{1}$ и $\phi_{2}$ рассчитываются по формулам (2) и (4): $\phi_{1}=0.9999943, \phi_{2}=0.9999979$.

Параметры стационарного режима определяются по формулам (11)-(14): $p_{12}^{\mathrm{st}}=0.0199161 ; p_{14}^{\mathrm{st}}=0.0199156 ; L_{1}^{\mathrm{st}}=3551227 ; L_{2}^{\mathrm{st}}=742628 ; L_{3}^{\mathrm{st}}=$ $=2546145 ; L_{4}^{\text {st }}=113160000$.

Параметры $a$ и $b$ определяются формулам (15): $a=0.693136, b=3.223092$.

Решение задачи Коши для системы дифференциальных уравнений (1)-(8) осуществлялось численно методом Рунге-Кутта 4-го порядка.

Предполагалось, что клеточная популяция находится в стационарном состоянии в момент гибели $20 \%$ зрелых клеток, поэтому начальные условия имеют следующий вид:

$$
\left.L_{1}\right|_{t \leqslant 0}=L_{1}^{\mathrm{st}},\left.\quad L_{2}\right|_{t \leqslant 0}=L_{2}^{\mathrm{st}},\left.\quad L_{3}\right|_{t \leqslant 0}=L_{3}^{\mathrm{st}},\left.\quad L_{4}\right|_{t<0}=L_{4}^{\mathrm{st}},\left.\quad L_{4}\right|_{t=0}=0.8 L_{4}^{\mathrm{st}} .
$$

На рис. 2-5 приводится изменение плотности клеток в клеточной популяции в разные этапы их жизненного цикла с момента гибели зрелых клеток в клеточной популяции. Правые графики дублируют информацию с левых графиков на более коротком интервале времени. Штриховая линия - плотность клеток на каждом этапе в стационарном состоянии клеточной популяции.

Из данных, представленных на рис. 2-5, видно, что после гибели части клеток происходит увеличение интенсивности пролиферативных процессов. В начальный период восстановления динамика плотности клеток в стадиях покоя, деления и роста носит характер периодических колебаний с постепенно уменьшающейся амплитудой. В итоге достигается нормальная плотность клеток, находящихся во всех стадиях, что соответствует представлениям о пролиферативном гомеостазе в нормальных клеточных популяциях.

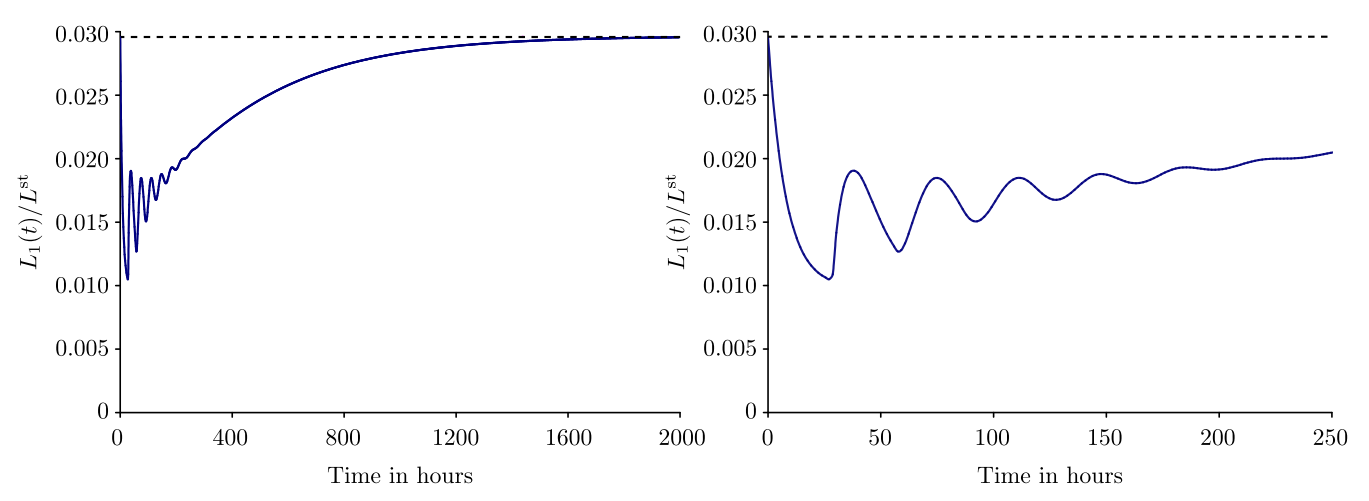

Рис. 2. Динамика плотности клеток в стадии покоя [Figure 2. The dynamics of the density of cells in the $\mathrm{G}_{0}$ phase] 

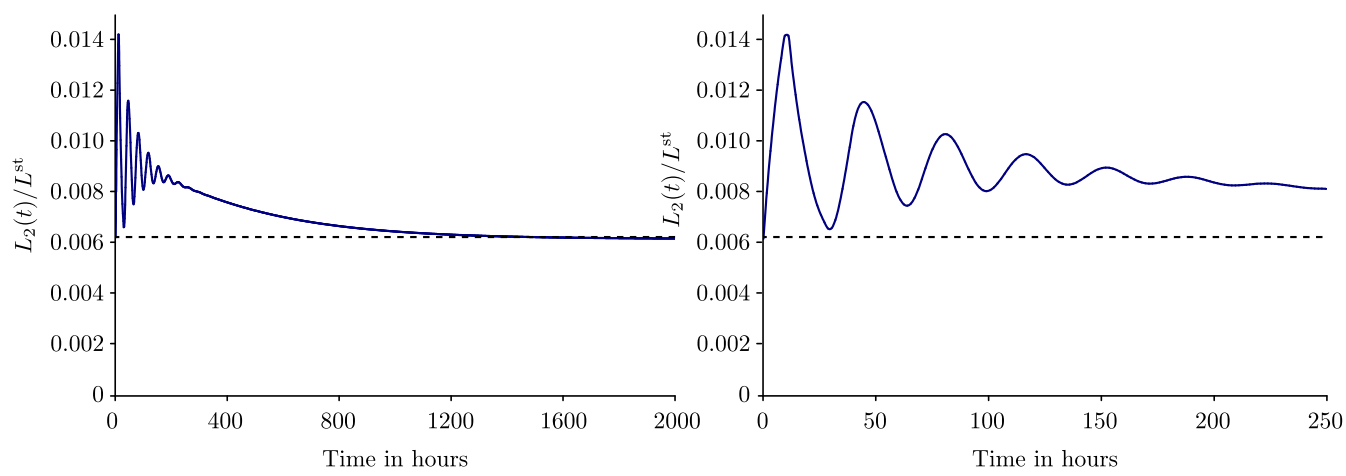

Рис. 3. Динамика плотности клеток в стадии деления

[Figure 3. The dynamics of the density of cells in the division stage]
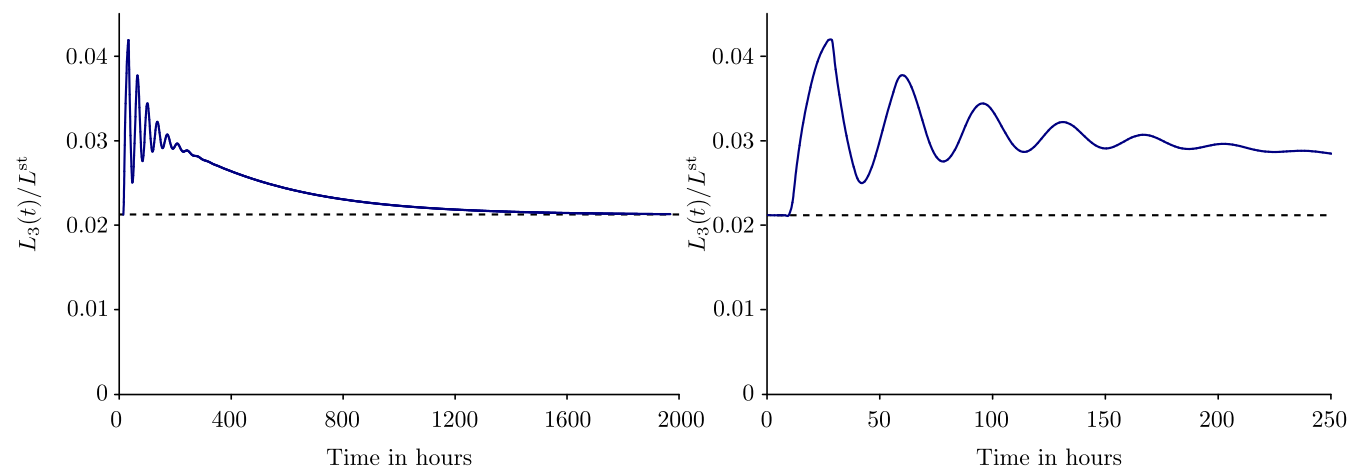

Рис. 4. Динамика плотности клеток в стадии роста

[Figure 4. The dynamics of the density of cells in the $\mathrm{G}_{1}$ phase]
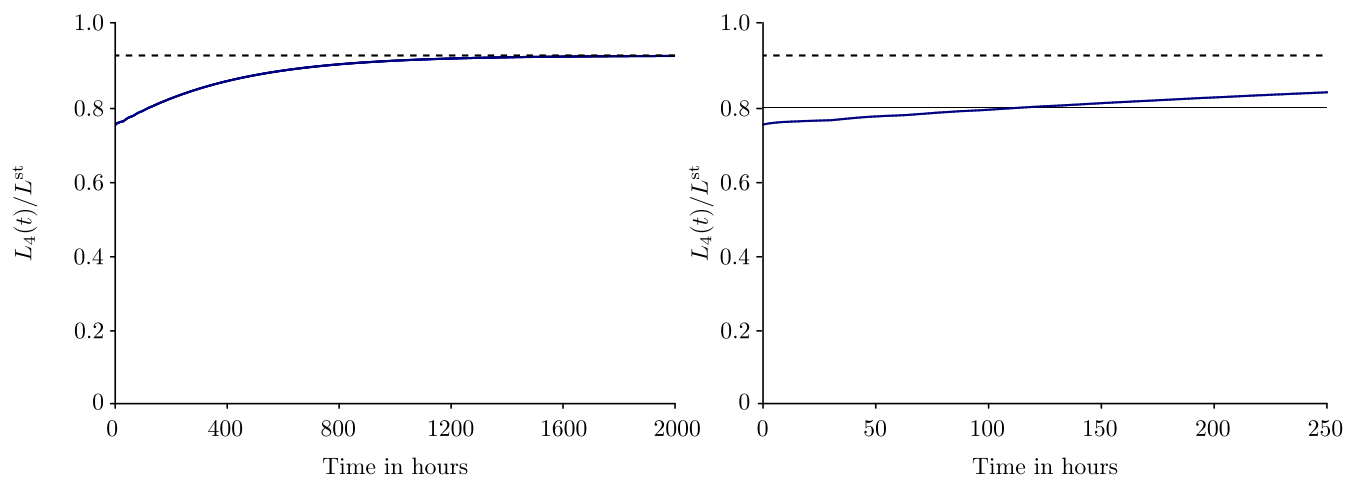

Рис. 5. Динамика плотности клеток в стадии зрелости

[Figure 5. The dynamics of the density of cells in the maturity stage] 
5. Сопоставление результатов вычислительного эксперимента с экспериментальными данными. В работе [36] приводятся результаты исследования влияния гамма-облучения на пролиферативные процессы в дуоденальном эпителии мышей. Облучение выполнялось однократно на всю поверхность тела. Известно, что кишечный эпителий обладает выраженной способностью к регенерации после повреждений за счет продукции новых клеток. В работе приводится динамика количества клеток в М- и S-фазах в течение 100-180 часов после облучения (в зависимости от дозы). Доза облучения варьировалась от $150 \mathrm{R}$ до $1000 \mathrm{R}$. В целом авторы работы пришли к следующим интересным выводам:

- облучение воздействует на все клетки, но степень чувствительности клеток зависит от того, в какой фазе находилась клетка в момент облучения; наиболее уязвимой является М-фаза;

- под воздействием облучения клетки блокируются в фазах $\mathrm{G}_{1}$ и $\mathrm{G}_{2}$, из-за чего наблюдается уменьшение числа клеток в M- и S-фазах непосредственно после облучения;

- в течение восстановительного периода динамика числа клеток в M- и Sфазах выглядит как «последовательность вершин и впадин», которая подобна флуктуациям, наблюдающимся и в тканях мышей, не подвергавшихся облучению; эксперименты показали, что такой характер изменений наблюдается при всех исследованных вариантах доз, но степень изменений зависит от дозы, а именно, возрастает при ее увеличении;

- со временем параметры интенсивности клеточного деления возвращаются в норму.

На рис. 6, 7 приведены экспериментальные данные по динамике числа клеток после облучения в дозах $600 \mathrm{R}$ и $750 \mathrm{R}$, на рис. 8 приводится общая схема, описывающая динамику клеток в S- и M-фазах (рисунки воспроизведены из работы [36]; верхние рисунки отражают динамику числа клеток в М-фазе (the number of mitotic figures per crypt), нижние рисунки - динамику числа клеток в S-фазе (the number of labeled nuclei per crypt); прерывистая линия динамика числа клеток в M- и S-фазах в тканях мышей, не подвергавшихся облучению).

Из представленных на рисунках данных видно, что в восстановительный период динамика числа клеток в фазах $\mathrm{M}$ и $\mathrm{S}$ носит характер затухающих колебаний, которые имели место и в нашем вычислительном эксперименте в сопоставимый по длительности начальный период времени после утраты части клеток. Это, на наш взгляд, свидетельствует об адекватности предложенной нами модели.

Однако стоит отметить и тот факт, что, по экспериментальным данным работы [36], число клеток в фазах $\mathrm{M}$ и $\mathrm{S}$ в кишечном эпителии и в нормальном состоянии носит характер колебаний с постоянной амплитудой, чего не наблюдается в нашем вычислительном эксперименте.

В целом экспериментальная работа [36] подтверждает нашу идею о том, что важно учитывать неоднородность клеточной популяции на разных этапах жизненного цикла при моделировании радиационного воздействия на опухолевые и нормальные ткани, т.к. в ней указывается, что степень чувствительности клеток к облучению зависит от того, в какой фазе находилась клетка в момент облучения. 

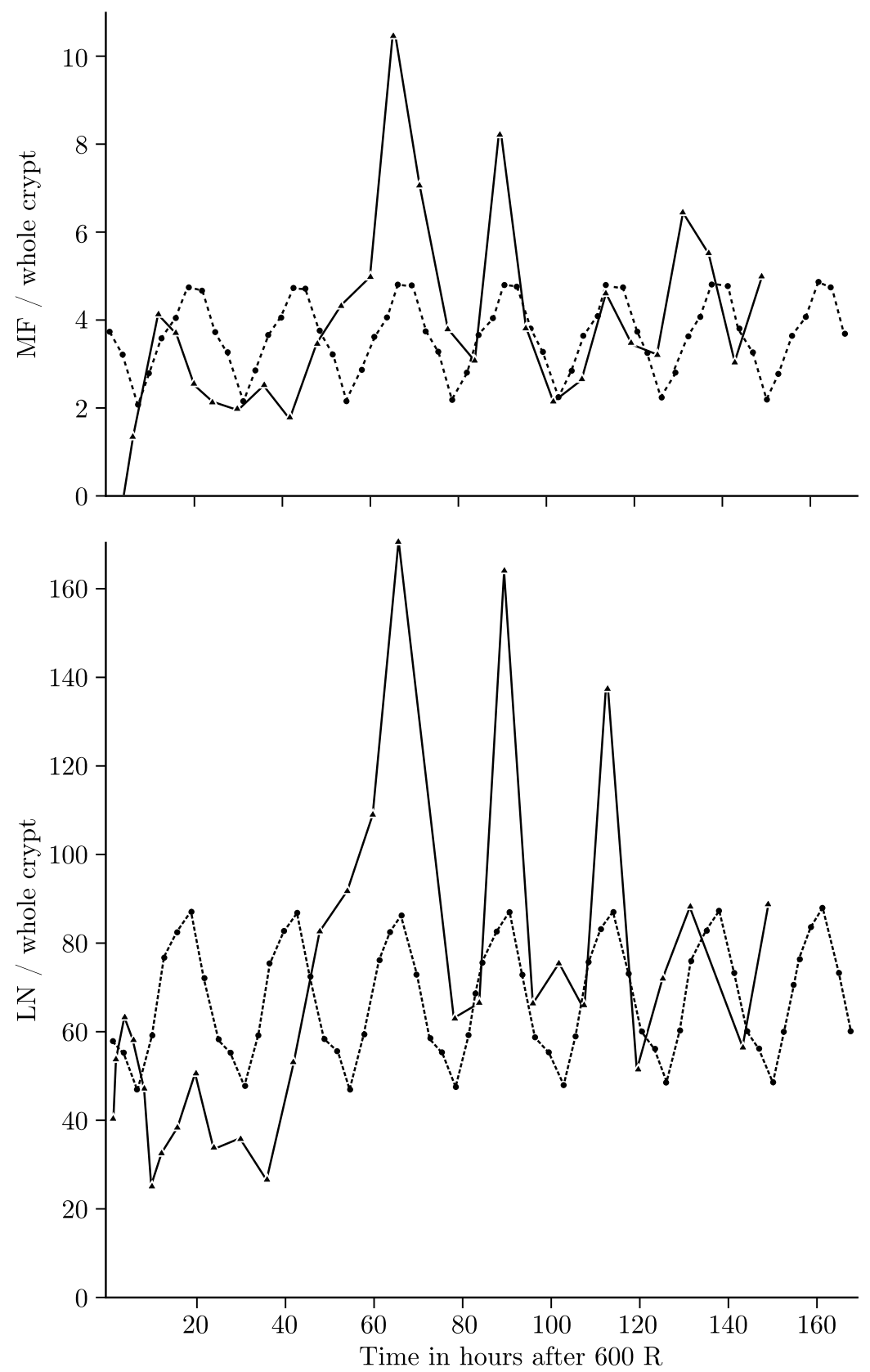

Рис. 6. Динамика числа клеток в М- и S-фазах после облучения при дозе $600 \mathrm{R}$

[Figure 6. Dynamics of the number of cells in the $\mathrm{M}$ (lower graph) and S (upper grap) phases after irradiation at a dose of $600 \mathrm{R}$. Solid lines connect experimental points; dotted lines connect control values (reproduced from [36])] 

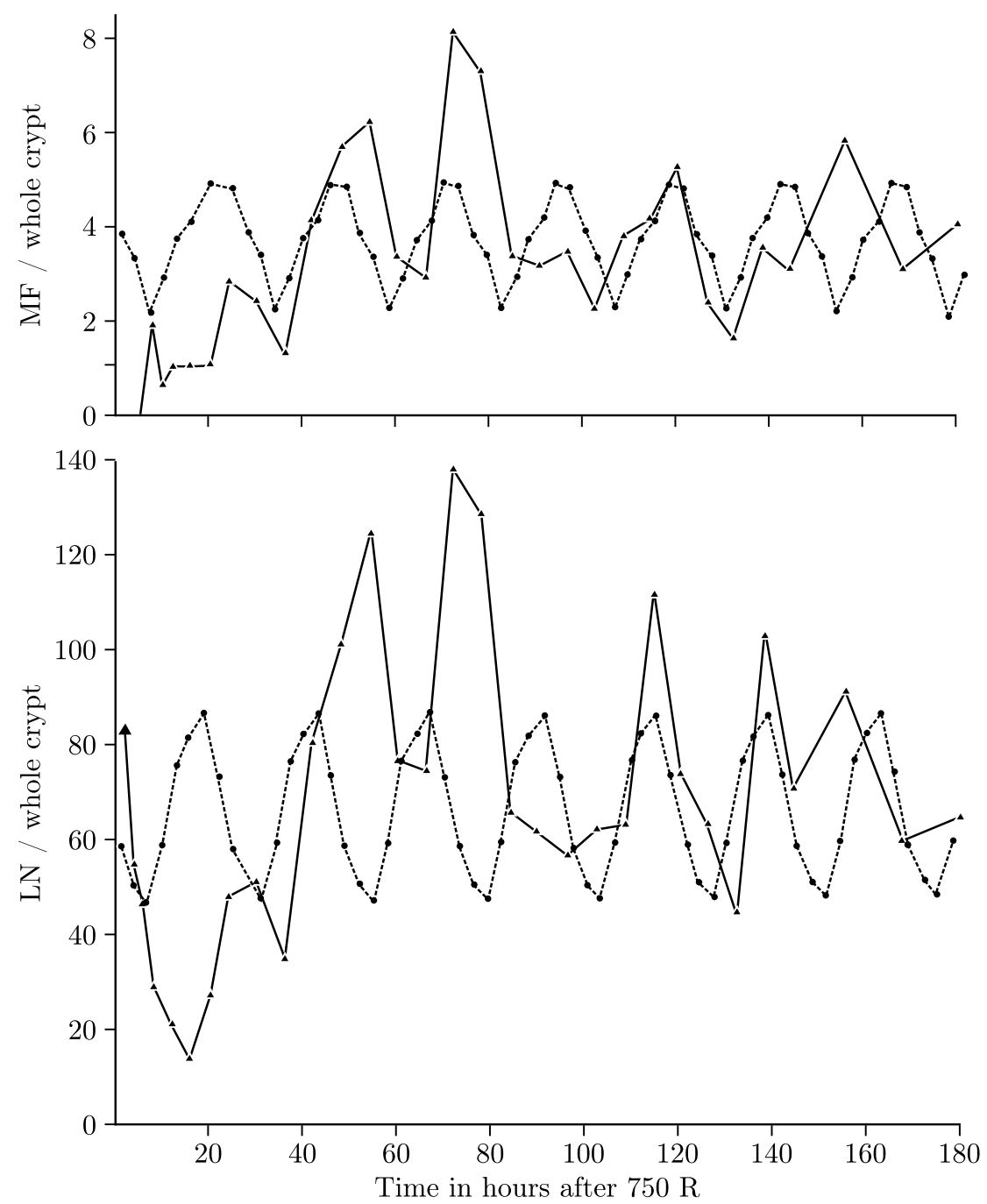

Рис. 7. Динамика числа клеток в М- и S-фазах после облучения при дозе $750 \mathrm{R}$ [Figure 7. Dynamics of the number of cells in the $M$ (lower graph) and S (upper grap) phases after irradiation at a dose of $750 \mathrm{R}$. Solid lines connect experimental points; dotted lines connect control values (reproduced from [36])] 

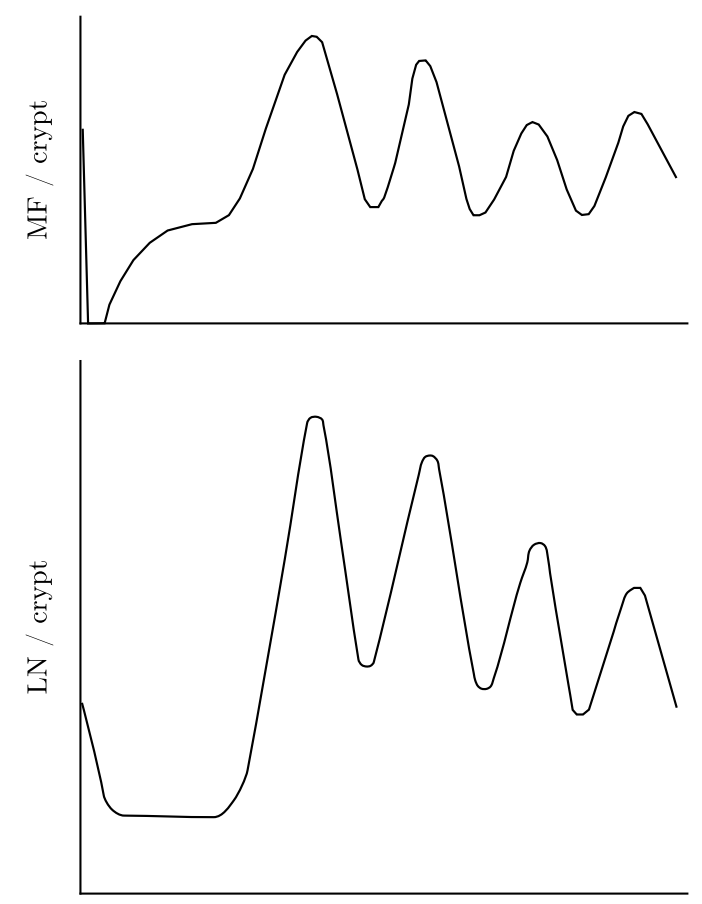

Time after irradiation

Рис. 8. Общая схема динамики числа клеток в М- и S-фазах после облучения

[Figure 8. The schematic diagram of changes in the number of cells in M (lower graph) and S (upper grap) phases after irradiation (reproduced from [36])]

Эта экспериментальная работа также позволяет выбрать направление, в котором необходимо модифицировать предложенную нами математическую модель, чтобы она более точно описывала влияние радиационного облучения на опухолевые и нормальные ткани: стадию деления следует разбить на отдельные фазы, чтобы можно было учитывать блокирование клеток в фазах $\mathrm{G}_{1}$ и $\mathrm{G}_{2}$ под воздействием радиации, а также зависимость чувствительности клеток к радиационному воздействию от фазы жизненного цикла.

Заключение. Предлагаемая модель пролиферативного гомеостаза в нормальной клеточной популяции разработана на основании представлений о жизненном цикле клетки и регуляции плотности клеток в популяции по принципу обратной связи таким образом, чтобы поддерживать необходимое количество зрелых клеток. Модель может быть использована как основа для построения модели опухолевого роста, в которой значения параметров, определяющих интенсивность пролиферативных процессов, отличаются от значений тех же параметров в нормальной популяции клеток, а также для моделирования воздействия различных видов лечения на нормальные и опухолевые ткани.

Конкурирующие интересы. Заявляем, что в отношении авторства и публикации этой статьи конфликта интересов не имеем.

Авторский вклад и ответственность. Все авторы принимали участие в разработке концепции статьи и в написании рукописи. Авторы несут полную ответственность за предоставление окончательной рукописи в печать. Окончательная версия 
рукописи была одобрена всеми авторами.

Финансирование. Исследование выполнялось без финансирования.

Благодарность. Авторы благодарны рецензентам за тщательное прочтение статьи и ценные предложения и комментарии.

\section{Библиографический список}

1. Latest global cancer data: Cancer burden rises to 18.1 million new cases and 9.6 million cancer deaths in 2018: Press Release no. 263. Lyon: IARC, 2018. 3 pp., http://www.iarc. $\mathrm{fr} / \mathrm{en} / \mathrm{media}$-centre/pr/2018/pdfs/pr263_E.pdf

2. Norton L. A Gompertzian Model of Human Breast Cancer Growth // Cancer Res., 1988. vol. 48, no. 24 (Part 1). pp. 7067-7071.

3. Hart D., Shochat E., Agur Z. The growth law of primary breast cancer as inferred from mammography screening trials data// Br. J. Cancer, 1998. vol.78. pp. 382-387. doi: 10 . 1038/bjc.1998.503.

4. de Vladar H., González J. Dynamic response of cancer under the influence of immunological activity and therapy // J. Theor. Biol., 2004. vol. 227, no. 3. pp. 335-348. doi: 10.1016/j . jtbi.2003.11.012.

5. de Pillis L., Radunskaya A., Wiseman C. A validated mathematical model of cell-mediated immune response to tumor growth// Cancer Res., 2005. vol.65, no.17. pp. 7950-7958. doi: 10.1158/0008-5472. can-05-0564.

6. Page K., Uhr J. Mathematical models of cancer dormancy // Leuk. Lymphoma, 2005. vol. 46, no. 3. pp. 313-327. doi : 10.1080/10428190400011625.

7. d'Onofrio A. A general framework for modeling tumor-immune system competition and immunotherapy: Mathematical analysis and biomedical inferences // Physica D: Nonlinear Phenomena, 2005. vol. 208, no. 3-4. pp. 220-235. doi: 10.1016/j.physd.2005.06.032.

8. Eftimie R., Bramson J. L., Earn D. J. D. Interactions Between the Immune System and Cancer: A Brief Review of Non-spatial Mathematical Models // Bull. Math. Biol., 2011. vol. 73, no. 1. pp. 2-32. doi: 10.1007/s11538-010-9526-3.

9. Grimes D. R., Kelly C., Bloch K., Partridge M. A method for estimating the oxygen consumption rate in multicellular tumour spheroids // J. Royal Soc. Interface, 2013. vol.11, no. 92, 20131124. doi: 10.1098/rsif.2013.1124.

10. Пономаренко Н. С., Книгавко В. Г., Батюк Л. В., Бондаренко М. А. Математическое моделирование распределения кислорода в злокачественных опухолях // Becmu Haицональной Академии Наук Беларуси. Серия Медичинских наук, 2015. №4. С. 61-67, http://repo.knmu.edu.ua/handle/123456789/11866.

11. Книгавко В. Г., Бондаренко М. А. Математическое моделирование диффузии и потребления кислорода в злокачественной опухоли // Биофизика, 2005. Т. 50, № 3. С. 544-549.

12. Coldman A. J., Goldie J. H. A model for the resistance of tumor cells to cancer chemotherapeutic agents // Math. Biosci., 1983. vol.65, no.2. pp. 291-307. doi: 10.1016/ 0025-5564 (83) 90066-4.

13. Iwasa Y., Nowak M. A., Michor F. Evolution of resistance during clonal expansion // Genetics, 2006. vol.172, no. 4. pp. 2557-2566. doi: 10.1534/genetics.105.049791.

14. Kimmel M., Swierniak A., Polanski A. Infinite-dimensional model of evolution of drug resistance of cancer cells // J. Math. Syst. Estim. Control, 1998. vol.8, no. 1. pp. 1-16.

15. Komarova N. Stochastic modeling of drug resistance in cancer// J. Theor Biol., 2006. vol. 239, no. 3. pp. 351-366. doi: 10.1016/j.jtbi.2005.08.003.

16. Tomasetti C., Levy D. An elementary approach to modeling drug resistance in cancer // Math. Biosci. Eng., 2010. vol. 7, no.4. pp. 905-918. doi: 10.3934/mbe.2010.7.905.

17. Cho H., Levy D. Modeling continuous levels of resistance to multidrug therapy in cancer // Appl. Math. Model., 2018. vol.64. pp. 733-751, arXiv: 1806.07557 [q-bio.PE]. doi: 10.1016/ j.apm.2018.07.025. 
18. Cho H., Ayers K., DePills L., Kuo Y.-H., Park J., Radunskaya A., Rockne R. Modeling acute myeloid leukemia in a continuum of differentiation states // Letters in Biomathematics, 2018. vol. 5, no. Suppl. 1. pp. S69-S98. doi: 10.1080/23737867.2018.1472532; doi:10.1101/ 2374382.

19. Greene J. M., Gevertz J. L., Sontag E. D. A mathematical approach to differentiate spontaneous and induced evolution to drug resistance during cancer treatment, 2018, https: //www. biorxiv.org/content/10.1101/235150v2. doi: 10.1101/235150.

20. Pisco A. O., Brock A., Zhou J., Moor A., Mojtahedi M., Jackson D., Huang S. NonDarwinian dynamics in therapy-induced cancer drug resistance// Nat. Commun., 2013. vol. 4, 2467. doi: 10.1038/ncomms3467.

21. Смирнова М. А. Радиация и организм млекопитающих: модельный подход. М., Ижевск: Регулярная и хаотическая динамика, 2006. 224 с.

22. Liu Z., Yang C. A Mathematical Model of Cancer Treatment by Radiotherapy // Comp. Math. Met. Med., 2014. vol. 2014, 172923. 12 pp. doi: 10.1155/2014/172923.

23. Сакович В. А., Смирнова О. А. Математическое моделирование влияние радиации на продолжительность жизни млекопитающих // Физика элементарных частиц и атомного ядра, 2003. Т. 134, №6. С. 1436-1484.

24. Matveev A. S., Savkin A. V. Optimal chemotherapy regimens: influence of tumours on normal cells and several toxicity constraints // IMA J. Math. Appl. Med. Biol., 2001. vol. 18, no. 1. pp. 25-40.

25. Shakhmurov V. B. On the dynamics of a cancer tumor growth model with multiphase structure, 2018, arXiv: 1801.05389 [q-bio.TO].

26. Shakhmurov V., Maharramov A., Shahmurzada B. The local and global dynamics of a cancer tumor growth and chemotherapy treatment model, 2018, arXiv: 1803.05330 [math.DS].

27. Diabate M., Coquille L., Samson A. Parameter estimation and treatment optimization in a stochastic model for immunotherapy of cancer, 2018, arXiv: 1806.01915 [q-bio.PE].

28. Baar M., Coquille L., Mayer H., Holzel M., Rogava M., Tüting T., Bovier A. A stochastic model for immunotherapy of cancer // Scientific Reports, 2016. vol. 6, 24169. doi: 10.1038/ srep24169.

29. Novozhilov A. S., Berezovskaya F. S., Koonin E. V., Karev G. P. Mathematical modeling of tumor therapy with oncolytic viruses: Regimes with complete tumor elimination within the framework of deterministic models // Biol. Direct, 2006. vol.1, no. 1, 6. 18 pp. doi: 10 . 1186/1745-6150-1-6.

30. Ганцев Ш. Х., Хуснутдинов Ш. М. Патология и морфологическая характеристика опухолевого роста. М.: Медицинское информационное агентство, 2003. 208 с.

31. Ганцев К. Ш. Улучшение нестандартных операций в абдоминальной онкологии: Дисс. ... доктора мед. наук. Уфа, 2005. 267 с.

32. Танюкевич (Франц) М. В. Модели и методы комплексных исследований медико-биологических процессов в онкологии: Дисс. ... канд. техн. наук. Уфа, 2005. 153 с.

33. Пехов А. П. Биология: медицинская биология, генетика и паразитология. М.: ГЕОТАР-Медиа, 2011. 656 с.

34. Мезен Н. И., Квачева З. Б., Сычик Л. М. Стволовые клетки. Минск: Белорус. гос. мед. ун-т, 2014. 62 с.

35. Baserga R. The Biology of Cell Reproduction. Cambridge, Mass.: Harvard University Press, 1985. $\mathrm{xi}+251 \mathrm{pp}$.

36. Lesher J., Lesher S. Effects of Single-Dose, Whole-Body, ${ }^{60}$ Co Gamma Irradiation on Number of Cells in DNA Synthesis and Mitosis in the Mouse Duodenal Epithelium // Radiation Research, 1970. vol.43, no. 2. pp. 429-438. doi: 10.2307/3573046. 


\title{
MSC: 92D25, 37N25, 34C60
}

\section{Tumor growth and mathematical modeling of system processes}

\author{
Sh. Kh. Gantsev ${ }^{1}$, R. N. Bakhtizin ${ }^{2}$, M. V. Frants $^{3}$, K. Sh. Gantsev ${ }^{1}$ \\ 1 Bashkir State Medical University, \\ 3, Lenin st., Ufa, 450000, Russian Federation. \\ 2 Ufa State Petroleum Technological University, \\ 1, Kosmonavtov st., Ufa, 450062, Russian Federation. \\ 3 Ufa State Aviation Technical University, \\ 12, K. Marx st., Ufa, 450008, Russian Federation.
}

\begin{abstract}
The paper deals with applying mathematical modeling to study tumor growth process and optimizing cancer treatment. A structured review of the studies devoted to this problem is given. The role of the cell life cycle in understanding the tumor growth and the mechanisms of cancer treatment is discussed. It is important that modern cancer treatment methods, in particular, chemotherapy and radiation therapy, affect both normal and tumor cells in certain stages of the life cycle and do not influence on cells in other stages. Cell life cycle description is given as well as the mechanisms that maintain and restore normal density of the cell population. A graph of cell life cycle stages and transitions is demonstrated. Dynamic mathematical model of proliferative homeostasis in the cell population is proposed, which takes into account the heterogeneity of cell populations by life cycle stages. The model is a system of differential equations with delays. The stationary state of the model is investigated, which allows to determine the parameters values for the normal cell population. The results of a numeric experiment is obtained, which is focused on the process of cell population density recovery after mass death of cells. As the experiment shows, after cell death, the densities of cells in different life cycle stages are restored to normal values, which corresponds to the concepts of proliferative homeostasis in cell populations.
\end{abstract}

Keywords: tumor growth, proliferative homeostasis, cell life cycle, cell kinetics.

\section{Research Article}

(ㅇ)(i) The content is published under the terms of the Creative Commons Attribution 4.0 International License (http://creativecommons.org/licenses/by/4.0/)

Please cite this article in press as:

Gantsev Sh. Kh., Bakhtizin R. N., Frants M. V., Gantsev K. Sh. Tumor growth and mathematical modeling of system processes, Vestn. Samar. Gos. Tekhn. Univ., Ser. Fiz.-Mat. Nauki [J. Samara State Tech. Univ., Ser. Phys. Math. Sci.], 2019, vol. 23, no. 1, pp. 131-151. doi: 10.14498/vsgtu1661 (In Russian).

\section{Authors' Details:}

Shamil Kh. Gantsev (D) https://orcid.org/0000-0003-2047-963X

Dr. Med. Sci., Professor; Head of Department; Dept. of Oncology; e-mail:prfg@mail.ru Ramil N. Bakhtizin; Dr. Phys. \& Math. Sci., Professor; Rector; e-mail:rektor@rusoil.net Marina V. Frants (1) https://orcid.org/0000-0002-5324-2463

Cand. Tech. Sci.; Associate Professor; Dept. of Business Economy; e-mail: tan-Marina@mail.ru Kamil Sh. Gantsev (D) https://orcid.org/0000-0002-7562-5684

Dr. Med. Sci.; Professor; Dept. of Oncology; e-mail: Gantseff@mail.ru 
Received: $18^{\text {th }}$ November, 2018 / Revised: $22^{\text {nd }}$ January, 2019 Accepted: $27^{\text {th }}$ January, $2019 /$ First online: $11^{\text {th }}$ March, 2019

Competing interests. We declare that we have no conflicts of interest in the authorship and publication of this article.

Authors' contributions and responsibilities. Each author has participated in the article concept development and in the manuscript writing. The authors are absolutely responsible for submitting the final manuscript in print. Each author has approved the final version of manuscript.

Funding. This research received no specific grant from any funding agency in the public, commercial, or not-for-profit sectors.

Acknowledgments. The authors are grateful to the referees for careful reading of the paper and valuable suggestions and comments.

\section{References}

1. Latest global cancer data: Cancer burden rises to 18.1 million new cases and 9.6 million cancer deaths in 2018, Press Release no. 263. Lyon, IARC, 2018, 3 pp., http://www.iarc. $\mathrm{fr} / \mathrm{en} / \mathrm{media}$-centre/pr/2018/pdfs/pr263_E.pdf

2. Norton L. A Gompertzian Model of Human Breast Cancer Growth, Cancer Res., 1988, vol. 48, no. 24 (Part 1), pp. 7067-7071.

3. Hart D., Shochat E., Agur Z. The growth law of primary breast cancer as inferred from mammography screening trials data, Br. J. Cancer, 1998, vol.78, pp. 382-387. doi: 10 . 1038/bjc.1998.503.

4. de Vladar H., González J. Dynamic response of cancer under the influence of immunological activity and therapy, J. Theor. Biol., 2004, vol.227, no.3, pp. 335-348. doi: 10.1016/j. jtbi.2003.11.012.

5. de Pillis L., Radunskaya A., Wiseman C. A validated mathematical model of cell-mediated immune response to tumor growth, Cancer Res., 2005, vol.65, no. 17, pp. 7950-7958. doi: 10.1158/0008-5472.can-05-0564.

6. Page K., Uhr J. Mathematical models of cancer dormancy, Leuk. Lymphoma, 2005, vol. 46, no. 3, pp. 313-327. doi: 10.1080/10428190400011625.

7. d'Onofrio A. A general framework for modeling tumor-immune system competition and immunotherapy: Mathematical analysis and biomedical inferences, Physica D: Nonlinear Phenomena, 2005, vol.208, no. 3-4, pp. 220-235. doi: 10.1016/j.physd.2005.06.032.

8. Eftimie R., Bramson J. L., Earn D. J. D. Interactions Between the Immune System and Cancer: A Brief Review of Non-spatial Mathematical Models, Bull. Math. Biol., 2011, vol. 73, no. 1, pp. 2-32. doi : 10.1007/s11538-010-9526-3.

9. Grimes D. R., Kelly C., Bloch K., Partridge M. A method for estimating the oxygen consumption rate in multicellular tumour spheroids, J. Royal Soc. Interface, 2013, vol. 11, no. 92, 20131124. doi: 10.1098/rsif.2013.1124.

10. Ponomarenko N. S., Knigavko V. G., Batyuk L. V.,Bondarenko M. A. Mathematical modeling of the oxygen distribution in malignant tumors, Vesti Natsional'noi Akademii Nauk Belarusi. Seriia Meditsinskikh nauk, 2015, no.4, pp. 61-67 (In Russian), http: //repo.knmu.edu.ua/handle/123456789/11866.

11. Knigavko V. G., Bondarenko M. A. Mathematical modeling of oxygen diffusion and consumption in malignant tumor, Biophysics, 2005, vol. 50, no. 3, pp. 479-483.

12. Coldman A. J., Goldie J. H. A model for the resistance of tumor cells to cancer chemotherapeutic agents, Math. Biosci., 1983, vol.65, no. 2, pp. 291-307. doi: 10.1016/0025-5564(83) 90066-4.

13. Iwasa Y., Nowak M. A., Michor F. Evolution of resistance during clonal expansion, Genetics, 2006, vol. 172, no. 4, pp. 2557-2566. doi: 10.1534/genetics.105.049791. 
14. Kimmel M., Swierniak A., Polanski A. Infinite-dimensional model of evolution of drug resistance of cancer cells, J. Math. Syst. Estim. Control, 1998, vol. 8, no. 1, pp. 1-16.

15. Komarova N. Stochastic modeling of drug resistance in cancer, J. Theor Biol., 2006, vol. 239, no. 3, pp. 351-366. doi: 10.1016/j.jtbi.2005.08.003.

16. Tomasetti C., Levy D. An elementary approach to modeling drug resistance in cancer, Math. Biosci. Eng., 2010, vol. 7, no. 4, pp. 905-918. doi: 10.3934/mbe.2010.7.905.

17. Cho H., Levy D. Modeling continuous levels of resistance to multidrug therapy in cancer, Appl. Math. Model., 2018, vol.64, pp. 733-751, arXiv: 1806.07557 [q-bio.PE]. doi : 10.1016/ j.apm. 2018.07.025.

18. Cho H., Ayers K., DePills L., Kuo Y.-H., Park J., Radunskaya A., Rockne R. Modeling acute myeloid leukemia in a continuum of differentiation states, Letters in Biomathematics, 2018, vol.5, no. Suppl. 1, pp. S69-S98. doi: 10.1080/23737867.2018.1472532; doi: 10 . $1101 / 2374382$.

19. Greene J. M., Gevertz J. L., Sontag E. D. A mathematical approach to differentiate spontaneous and induced evolution to drug resistance during cancer treatment, 2018, https: //www.biorxiv.org/content/10.1101/235150v2. doi: 10.1101/235150.

20. Pisco A. O., Brock A., Zhou J., Moor A., Mojtahedi M., Jackson D., Huang S. NonDarwinian dynamics in therapy-induced cancer drug resistance, Nat. Commun., 2013, vol. 4, 2467. doi: 10.1038/ncomms3467.

21. Smirnova O. A. Environmental Radiation Effects on Mammals. A Dynamical Modeling Approach. Cham, Springer, 2017, xxiii+359 pp. doi: 10.1007/978-3-319-45761-1.

22. Liu Z., Yang C. A Mathematical Model of Cancer Treatment by Radiotherapy, Comp. Math. Met. Med., 2014, vol. 2014, 172923, 12 pp. doi: 10.1155/2014/172923.

23. Sakovich V. A., Smirnova O. A. Modeling radiation effects on life span of mammals, Physics Particles and Nuclei, 2003, vol. 34, no. 6, pp. 743-766.

24. Matveev A. S., Savkin A. V. Optimal chemotherapy regimens: influence of tumours on normal cells and several toxicity constraints, IMA J. Math. Appl. Med. Biol., 2001, vol. 18, no. 1, pp. 25-40.

25. Shakhmurov V. B. On the dynamics of a cancer tumor growth model with multiphase structure, 2018, arXiv: 1801.05389 [q-bio.TO].

26. Shakhmurov V., Maharramov A., Shahmurzada B. The local and global dynamics of a cancer tumor growth and chemotherapy treatment model, 2018, arXiv: 1803.05330 [math.DS].

27. Diabate M., Coquille L., Samson A. Parameter estimation and treatment optimization in a stochastic model for immunotherapy of cancer, 2018, arXiv: 1806.01915 [q-bio.PE].

28. Baar M., Coquille L., Mayer H., Holzel M., Rogava M., Tüting T., Bovier A. A stochastic model for immunotherapy of cancer, Scientific Reports, 2016, vol.6, 24169. doi:10.1038/ srep24169.

29. Novozhilov A. S., Berezovskaya F. S., Koonin E. V., Karev G. P. Mathematical modeling of tumor therapy with oncolytic viruses: Regimes with complete tumor elimination within the framework of deterministic models, Biol. Direct, 2006, vol. 1, no.1, 6, 18 pp. doi: 10 . 1186/1745-6150-1-6.

30. Gantsev Sh. Kh., Khusnutdinov Sh. M. Patologiia i morfologicheskaia kharakteristika opukholevogo rosta [Pathology and morphological characteristics of tumor growth]. Moscow, Medical Information Agency, 2003, 208 pp. (In Russian)

31. Gantsev K. Sh. Improvement of non-standard operations in abdominal oncology, DMedSc Thesis. Ufa, 2005, 267 pp. (In Russian)

32. Tanyukevich (Frants) M. V. Models and methods of comprehensive studies of biomedical processes in oncology, Cand. Tech. Sci. Thesis. Ufa, 2005, 153 pp. (In Russian)

33. Pekhov A. P. Biologiia: meditsinskaia biologiia, genetika i parazitologiia [Biology: Medical Biology, Genetics, Parasitology]. Moscow, GEOTAR-Media, 2011, 656 pp. (In Russian)

34. Mezen N. I., Kvacheva Z. B., Sychik L. M. Stvolovye kletki [Stem Cells]. Minsk, Belarus State Medical Univ., 2014, 62 pp. (In Russian) 
35. Baserga R. The Biology of Cell Reproduction. Cambridge, Mass., Harvard University Press, $1985, \mathrm{xi}+251 \mathrm{pp}$.

36. Lesher J., Lesher S. Effects of Single-Dose, Whole-Body, ${ }^{60}$ Co Gamma Irradiation on Number of Cells in DNA Synthesis and Mitosis in the Mouse Duodenal Epithelium, Radiation Research, 1970, vol.43, no. 2, pp. 429-438. doi: 10.2307/3573046. 